\title{
The Watermark Technology Application Research Guided by the Idea of Computer Safe Operation
}

\author{
Juan $\mathrm{ZHONG}^{1, \text { a }}$, Hua LIAO \\ ${ }^{1}$ Gannan Medical University, Ganzhou 341000,China \\ ${ }^{2}$ Jiangxi Ganzhou power supply company, Ganzhou 341000,China \\ ajuanzhong@126.com
}

\begin{abstract}
Keywords: Watermark technology; Computer security; Digital watermarking; Digital copyright
\end{abstract}
\begin{abstract}
Electronic information technology in the construction and development of modern society in various fields and trades in occupies the important position and role. Electronic information technology application is based on the computer, but because of various kinds of information in the network virus viral, causing a computer security protection become one of the key technical problems. In recent years, the watermarking technology in the widely used in computer security, and achieved ideal effect and function. This paper systematically discusses the research and development of digital watermarking technology, first of all introduced and classification of information hiding technology, then analyses the model of digital watermarking technology, finally, the digital watermarking technology and its application prospect of the development and research of some possible directions are pointed out.
\end{abstract}

\section{Introduction}

With the rapid development of Internet technology and multimedia technology, digital information can be in the form of different transmission easily and quickly on the Internet. Multimedia communication has become an important means of communication between people. People communicate all kinds of information through the network, online trade, etc. Therefore, information security and confidentiality is more and more important. Security and confidentiality of information not only with the country's political, military and foreign affairs have a significant relationship, and with the country's economic, business and individuals shall have a great relationship. With the coming of information society, digital information and network has become an important part of people's life, they bring us convenient while, also brought us a hazard: sensitive information can easily be theft, tampering, illegal copying and dissemination, etc. So the information security has become the focus of people care about, is also the research hotspot and difficulty today [1-2].

At home and abroad, the watermarking technology widely used in computer security, cryptographic algorithms used by computer for the transmission of data encryption, and automatically generate a testing information, and the original to send at the same time, the automatic generation of test information is similar to real signature or seal, so that the recipient of its verification, the original judgment authenticity, the purpose is to provide data integrity protection and function of anti-denied, and fully guarantee the security of the computer, prevent malicious information dissemination and harassment. The important means of digital watermarking for copyright protection, has been widely research and application.

\section{Watermark technology development situation}

With the rapid development of multimedia information processing technology and the Internet, various forms of multimedia works, such as audio, video, animation, images, etc., are released through the network [3]. Because digital products has the issue of low cost, can be rapidly and copy etc., so get more and more widely used, which makes the data exchange and transmission becomes a relatively simple and efficient process. At the same time also have to face the threat of illegal use and spread. Any digital information and analog information, has its inherent error range, known as 
noise. The making process of the digital watermarking can be thought of as attach information such as property right as the noise of the integration in the original digital products; each digital watermarking system contains at least two components: the watermark embedded unit, the watermark detection and extraction unit. A diagram in figure 1 is a system of digital watermarking technology.

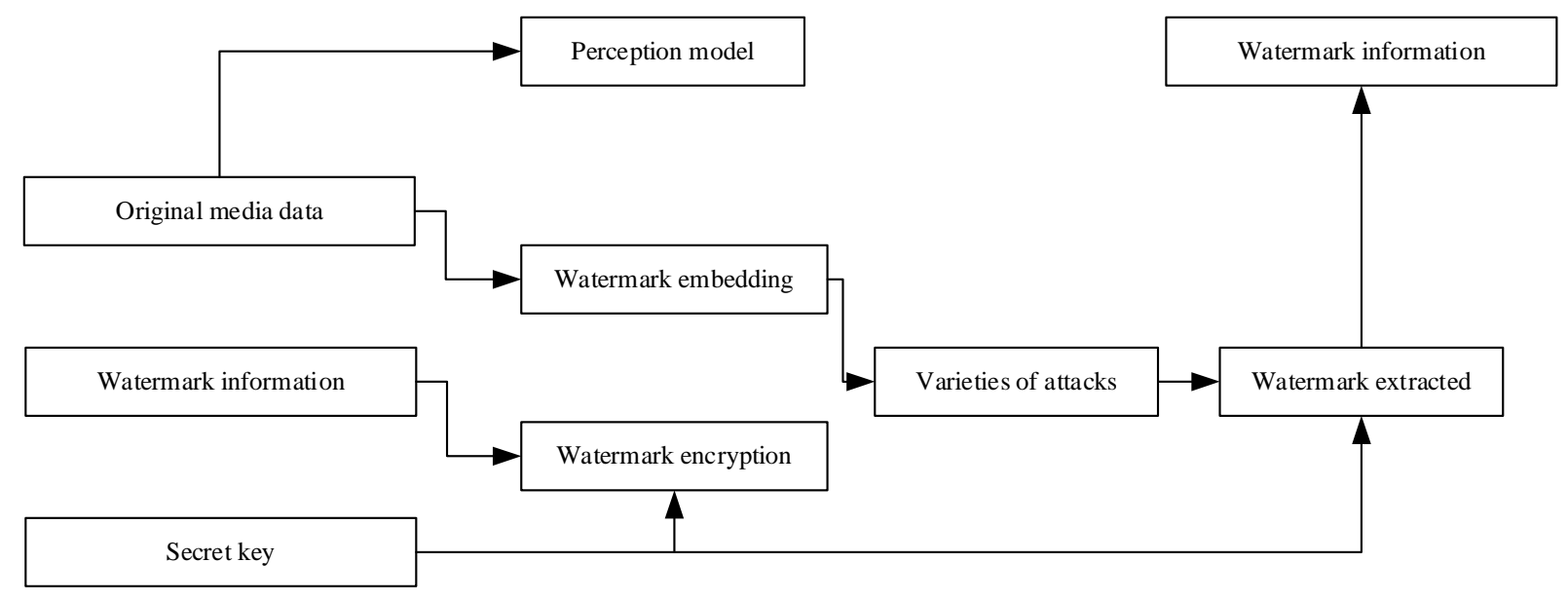

Figure 1. Digital watermarking system structure

With digital products of intellectual property protection problem increasingly dash forward show, digital watermark technology is developed rapidly under this application needs. Digital watermarking technology is through a certain algorithm to embed the iconic information directly multimedia content, but does not affect the value of the original content and use, and cannot be sensory systems perceive or noticed that only through a dedicated detector or reader can extract [4]. The watermark can be the author's serial number, company logo, has the special meaning of the text, etc., can be used to identify the sources and versions of documents, images or music products, the author, the owner, the issuer, the ownership of the legitimate users of digital products.

\section{The basic principle of digital watermarking technology}

Digital watermark is the effective method to realize copyright protection, has become a hot topic in the field of multimedia information security research, and is also the letter $\mathrm{J}$ important branch in the field of interest and hiding technology research. It through embedding secret information - the watermark in the raw data to confirm the ownership of data. By embedding the watermark can be a paragraph of text, logo, serial number, etc. Watermark is usually not visible or not, it has to do with the original data (such as images, audio, video, data, etc.) together and hidden, known as the integral part of the source data, and to experience some does not destroy the source data use value or the operation of the commercial value and survive. From the perspective of image processing, embedded watermarking signal can be regarded as superposition of a weak signal under strong background, as long as the superposition of the watermark signal strength is lower than the contrast threshold of HVS by space, time and frequency characteristics of the visual system [5].

Therefore, the use of human visual redundancy or insensitivity, image of its own uniqueness (individual image characteristics), data redundancy, the time-frequency local features, the creators of information and personal logo into the multimedia data, that people couldn't from perception to join the information on the surface, only dedicated test or computer software can detect the hidden information, so as to achieve the protection of intellectual property rights, the protection of copyright and other purposes. All methods of embedding watermark module contains the basic construction, that is, a watermark is embedded system and a watermark recovery system, and the watermark embedded system is shown in figure 2. 


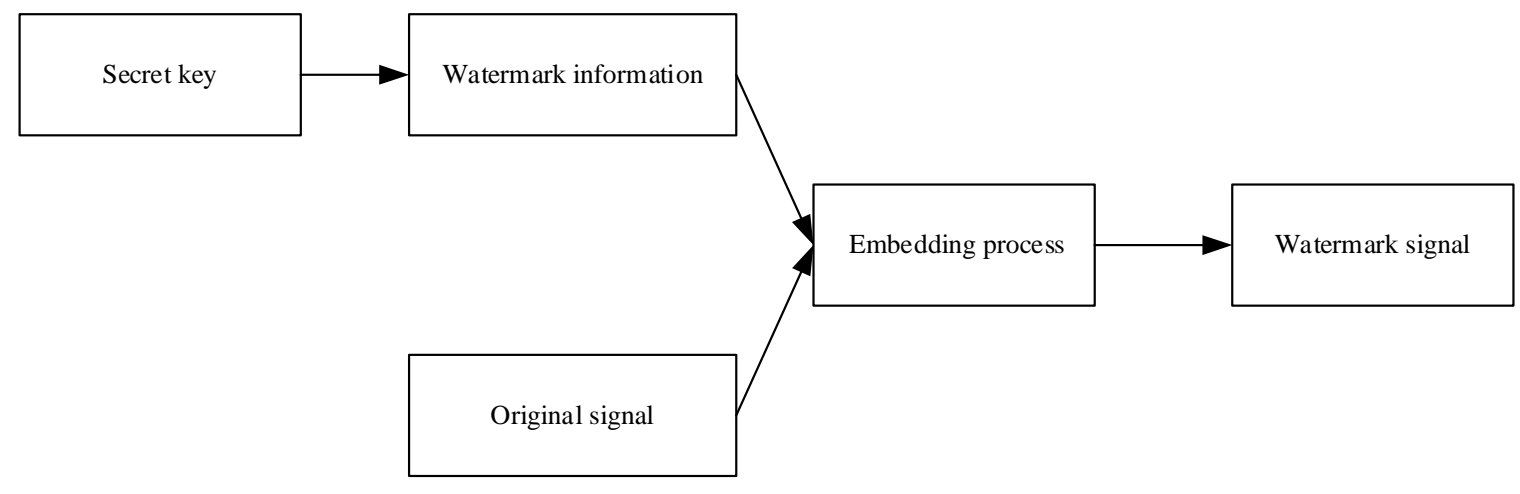

Figure 2. Watermark embedding process

One key can be used to strengthen safety, avoid unauthorized party back and modify the watermark, the watermark recovery system is shown in figure 3.

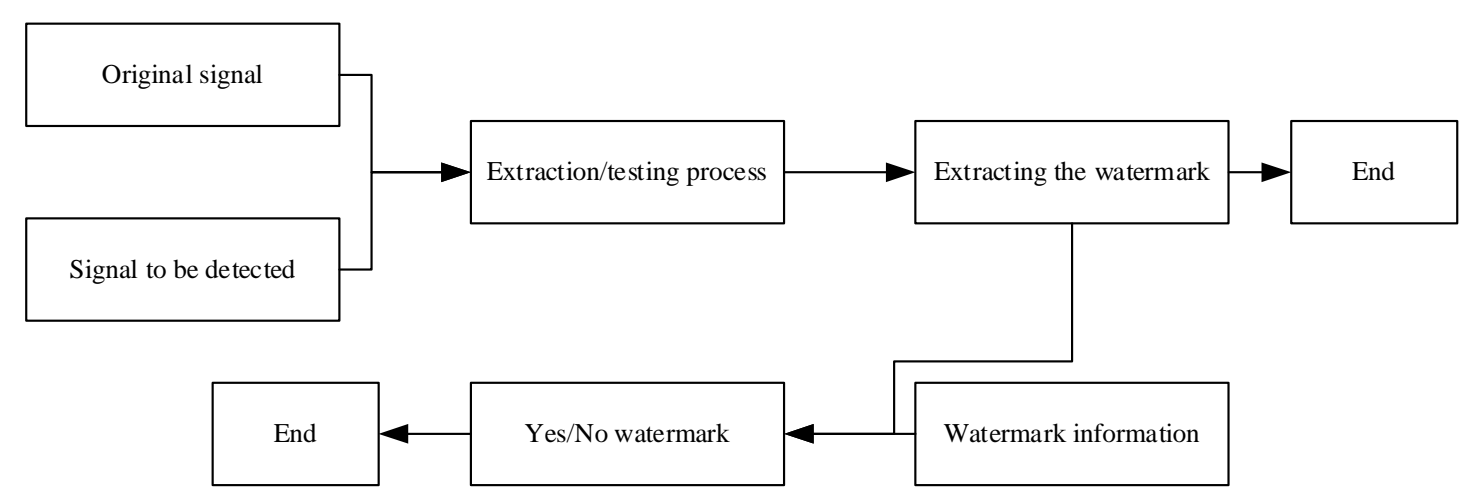

Figure 3. Watermark signal detection/extraction process

Digital watermarking technology besides should have the general characteristics of information hiding technology, and its inherent characteristics and research methods. For example, a word, from the view of the confidentiality of information security and the hidden information if is eroded, system can be regarded as safe, because the secret information not: but, in the digital watermarking system, the loss of the hidden information means that copyright information, lost, and lose the function of copyright protection, this system is a failure. Therefore, digital watermarking technology is usually in addition to should have the general characteristics of information hiding technology, with its own characteristics and research methods, namely has stronger robustness, security and transparency.

\section{The application of watermarking technique in the digital library guided by the idea of computer safe operation}

Digital library is the computer technology, communication technology and Internet technology is widely used as a result, is an important part of a national information infrastructure. Database development and utilization has become the core content of digital library information resource construction. But due to the openness and sharing database, database products infringed, the phenomenon such as piracy and random tampering with ongoing, correctly how to coordinate the database developers, users, the relationship between the copyright person, how to protect the intellectual property of digital library database is remarkable.

Watermark as a kind of information hiding technology, for the copyright protection of digital library, provides an effective solution. Database watermarking technology is used in digital signal processing method of multimedia database embedded in the hidden and difficult to detect markers, only through a dedicated detector or only with the owner of the corresponding key access to copyright watermark extraction or removed. Use of database watermarking technology can be the 
owner name, writing time, such as user ID symbol database owner or rights of a legitimate user works copyright information into the database, and join key, to protect the security of digital library database. Figure 4 is application of copyright protection for digital library database watermark model.

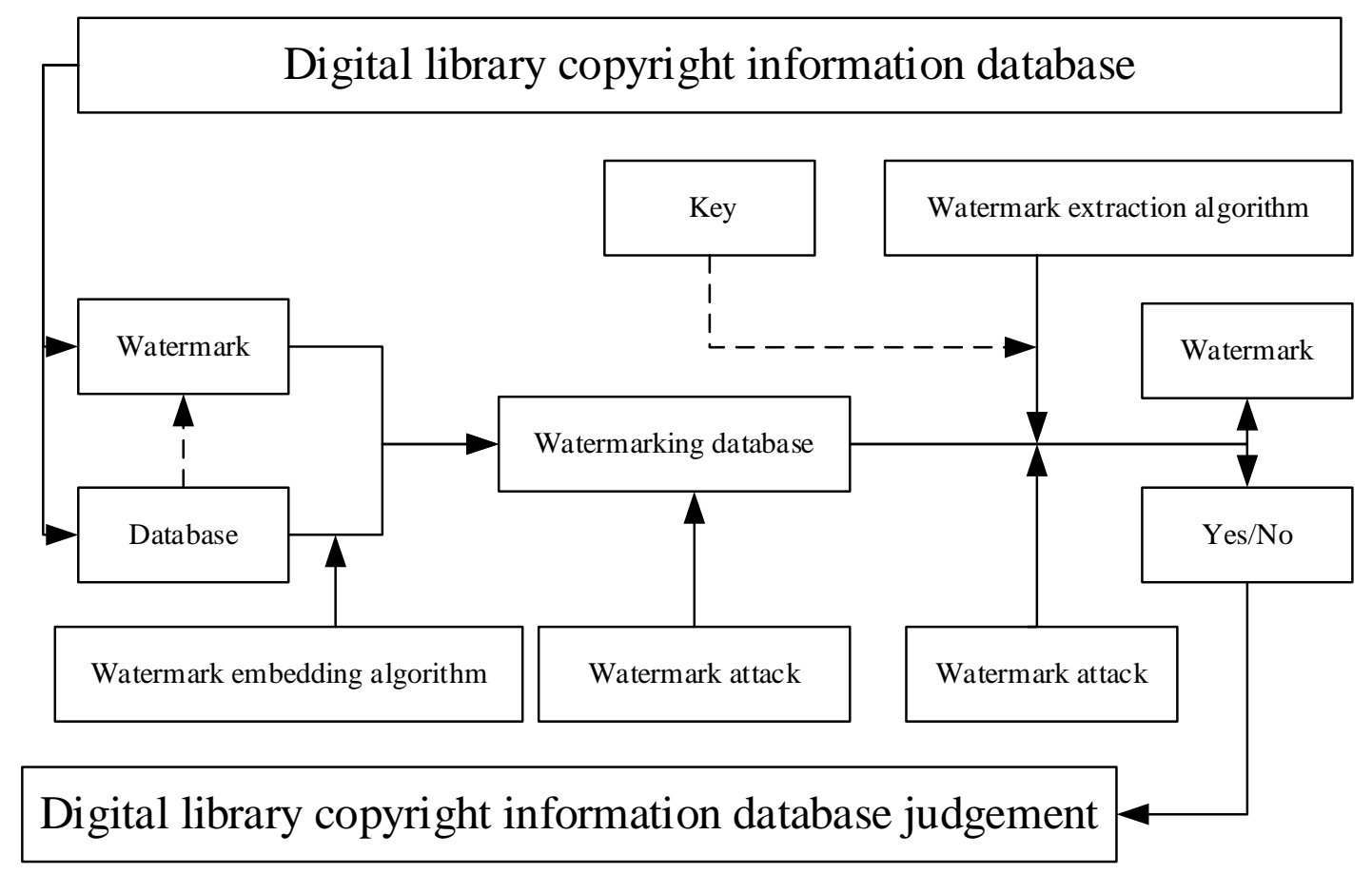

Figure 4.The application of watermarking technique in the digital library

\section{Conclusion}

With the increasing of the modern network environment management standard, in the development of electronic information technology, computer security has gradually become the focus of the world network technology industry focus on one of the problems. At home and abroad, the watermarking technology widely used in computer security, cryptographic algorithms used by computer for the transmission of data encryption processing, its purpose is to provide data integrity protection and function of anti-denied, and fully guarantee the security of the computer, prevent malicious information dissemination and harassment. In this paper, the development situation and technical principle of watermark technology into the in-depth research, discusses the computer guided by the idea of safe operation of the watermarking technology in the application of digital library, have important practical significance.

\section{References}

[1] Hehuan P, Li W, Xinxia R:Computer Science \& Education (ICCSE), 2012 7th International Conference on. IEEE, 2012: 610-612.

[2] Zhou Z, Xie S S, Chen D: Fundamentals of Digital Manufacturing Science. Springer London, 2012: 291-336.

[3] Schindler V, Roček A: Interfaces, 2013, 551: 64.

[4] Khan A I, Alam M M, Khan U A: International Journal of Computer Applications, 2013, 67(9).

[5] Xia W U: Canadian Social Science, 2013, 9(2): 34-39. 